\title{
Kapillarrohrmattensystem zur effektiven Nutzung von Solarenergie und Erdwärme
}

\author{
Siegfried Rolle, Roy-Oliver Adams, Marko Friedemann, Henner Hoppe, Michael Jergović
}

\section{Vorbetrachtungen zur Energieeinsparung}

Mit nahezu $30 \%$ Endenergieverbrauch liegen die Haushalte in gleicher Größenordnung, wie der Verkehr oder die Industrie und sind damit ein bedeutender Faktor bei allen Energiebetrachtungen. Zwei Drittel dieser Energie werden zu Heizzwecken eingesetzt. [1] Fortschritte bei der Nutzung alternativer Energien insbesondere $\mathrm{CO}_{2}-$ neutraler Systeme und energiesparender Technologien sind daher von großem Interesse.

In diesem Artikel wird eine Möglichkeit der Nutzung von Solarwärme unter Einbeziehung von saisonalen Speichern beschrieben. Der Einsatz eines flüssigkeitsdurchströmten Kapillarrohrmattensystems aus Kunststoff in Gebäuden ermöglicht eine Reihe effektiver Lösungen zum Wärmeübergang sowohl zur Kühlung als auch zur Heizung. Das Gesamtsystem thermischer Solarkollektor, Wärmeübergang (Heizung/Kühlung), Wärmepumpe und saisonaler Speicher im Gebäude bietet gute Bedingungen für ein energiesparendes Temperaturmanagement.

Vorausgesetzt werden alle Maßnahmen zur Energieeinsparung am Gebäude gemäß der 2. Novellierung der Wärmeschutzverordnung von 1995, die technisch gegenwärtig relativ unproblematisch umgesetzt werden können.

\section{Energiekonzept}

\subsection{Beschreibung des Installationsbeispiels}

Die Ausfuihrung eines Installationsbeispiels wurde in einem Musterhaus (Fertigteilhaus der Firma Ekofors Fabriks AB, Schweden, Standort: Oranienburg) realisiert, welches im folgenden beschrieben wird. Auf bautechnische Einzelheiten soll hier nicht eingegangen werden.

Die wichtigsten Daten des Gebäudes:

4 Personenhaushalt

Dachfläche:
Wohnfläche:

Außenfassade:

\section{Erdgeschoß}

Dachgeschoß

Fensterfläche:

Dachneigung:

$$
\begin{aligned}
& 95 \mathrm{~m}^{2} \\
& \approx 90 \mathrm{~m}^{2} \\
& \approx 39 \mathrm{~m}^{2} \\
& \approx 42 \mathrm{~m}^{2} \\
& \approx 120 \mathrm{~m}^{2} \\
& 45^{\circ}
\end{aligned}
$$

k-Werte ausgewählter Bauelemente:

Außenwand Erdgeschoß

Giebelseite Dachgeschoß

Dach

Fenster, Fenstertüren

Haustiir
$0,25 \mathrm{~W} / \mathrm{m}^{2} \mathrm{~K}$ $0,23 \mathrm{~W} / \mathrm{m}^{2} \mathrm{~K}$ $1,50 \mathrm{~W} / \mathrm{m}^{2} \mathrm{~K}$ $1,00 \mathrm{~W} / \mathrm{m}^{2} \mathrm{~K}$ $0.25 \mathrm{~W} / \mathrm{m}^{2} \mathrm{~K}$
Der Wandaufbau besteht im wesentlichen aus einem außenliegenden Klinkermauerwerk mit darunterliegender Holz/Mineralwolle-Sandwichwand.

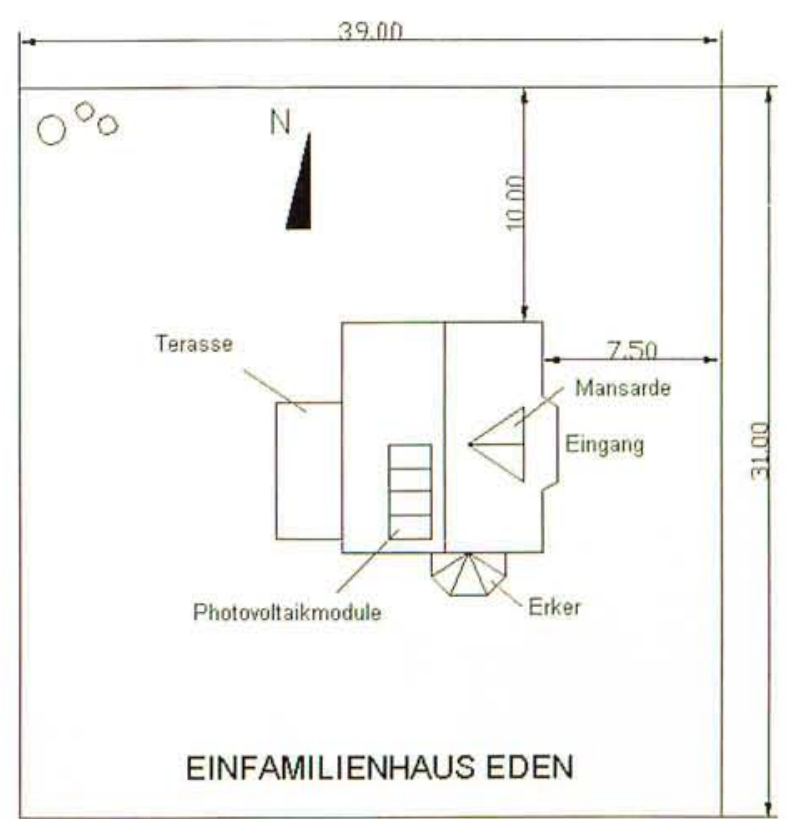

Bild 1: Lageskizze mit örtlichen Gegebenheiten des Musterhauses

\subsection{Basiskomponenten}

\subsubsection{Kapillarrohrmattensystem}

Zum Einsatz kommt das flüssigkeitsdurchströmte Kapillarrohrsystem CLINA ${ }^{\oplus}$ der Firma Clina Heiz- und Kühlelemente GmbH Berlin, welches in Form von Matten aus Polypropylen hergestellt wird. Dieses System wird insbesondere zum Kühlen von Industriegebäuden über 15 Jahre angewendet.

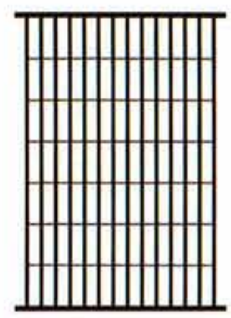

G - Matte

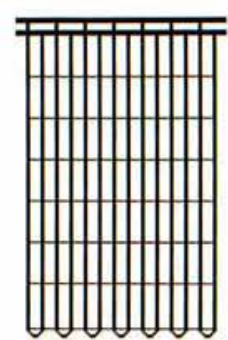

S - Matte

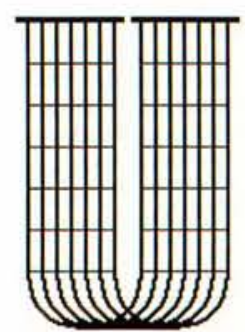

$\mathrm{U}$ - Matte
Zwischen zwei Stammrohren (Vor- und Rücklauf) mit einem Durchmesser von $20 \mathrm{~mm}$ befinden sich die Kapillarröhrchen (Außendurchmesser 3,4 mm, Innendurch- 
messer 2,3 mm) in verschiedenen Verlegearten (Kapillarrohrmattentypen, siehe Bild 2). Die Abstände der Röhrchen sind vom Mattentyp abhängig und können auch individuell variiert werden. Die maximale Kapillarrohrlänge sollte aus strömungstechnischen Gründen $12 \mathrm{~m}$ nicht ïberschreiten.

Das Kapillarrohrmattensystem ist äußerst flexibel und kann den verschiedenen Gegebenheiten der Einsatzorte sehr gut angepaßt werden. Durch die geringen Einbauhöhen (ca. $30 \mathrm{~mm}$ an den Sammlern bzw. ca. $10 \mathrm{~mm}$ an den Kapillaren) können die Matten im Fußboden, in der Decke, an Wänden und im Dachbereich angebracht werden.

Die relativ geringen Abmessungen der Vorlauf- und Rücklaufkapillare ermöglichen eine höhere Anzahl von Kapillaren pro Fläche im Mattensystem anzuordnen. Dadurch ergibt sich ein sehr günstiges Verhältnis von Wärmeübergangsfläche zu bedeckter Gesamtfläche bei minimaler Baudicke. Für Standardmatten (siehe Bild 3) beträgt dieses Verhältnis $0,74(74 \%)$ im Vergleich zu 0.40 (40\%) bei konventionellen Fußbodenheizungswerten von (siehe Bild 4).

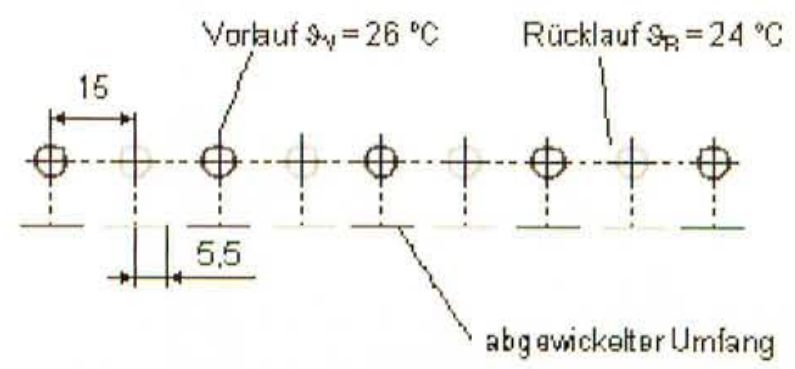

Bild 3: Wärmeübergangsflächen beim Kapillarrohrmattensystem (Schnitt, schematisch)

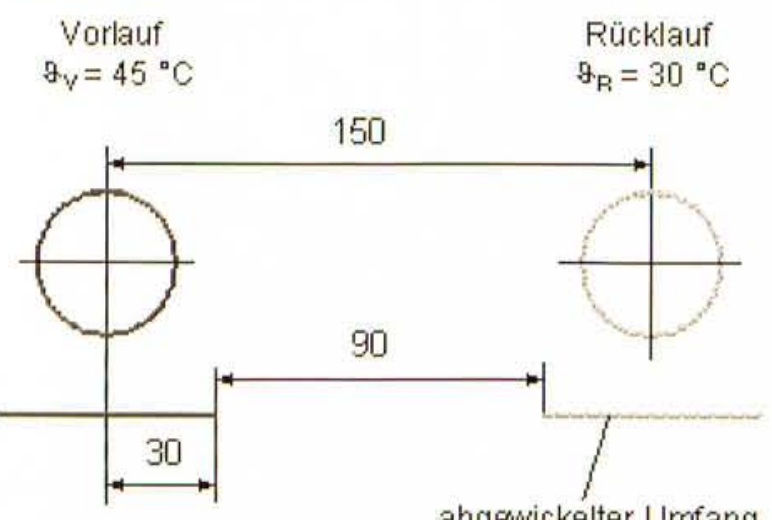

Bild 4: Wärmeübergangșflächen bei einer konventionellen Fußbodenheizung (Schnitt, schematisch)

Durch diese vorteilhaften Flächendaten kann im Fall der Heizung mit diesem System die Vorlauftemperatur gesenkt werden. Die Nutzung niedriger Wärmepotentiale, wie sie z. B. im Erdreich vorhanden sind, kommt so in den Bereich der Wirtschaftlichkeit. Für eine Wohnraumerwärmung auf $20^{\circ} \mathrm{C}$ bei angenommener Außentemperatur von $-14{ }^{\circ} \mathrm{C}$ reicht unter den gegebenen Bedingungen und einer Fußbodenverlegung im gesamten Raum eine Vorlauftemperatur von ca. $30{ }^{\circ} \mathrm{C}$. Bei Standardfußbodenheizungen liegt dieser Wert bei ca. $45^{\circ} \mathrm{C}$.

Für den Betrieb des Kapillarrohrmattensystems ist die korrosionsfreie Auslegung des Flüssigkeitskreislaufes zu beachten. Korrosionsprodukte würden zu Verunreinigungen in der Flüssigkeit führen, die die Kapillaren verstopfen können. Im Leitungsbereich können Kunststoffe eingesetzt werden. Die Armaturen (z. B. Pumpen) sollten aus Bronze bestehen. Mit einem geringen Aufpreis ist diese Anforderung realisierbar. Sollten Kapillaren beschädigt werden, z. B. bei Arbeiten am Fußboden oder an den Wänden, werden die betroffenen Stellen verschweißt. Diese zwar dauerhaft ausfallende Kapillare, beeinflußt das Gesamtsystem aber praktisch nicht, da nur ein kleiner Flächenanteil betroffen ist.

Im betrachteten Anwendungsfall wurden die Kapillarrohrmatten wie folgt installiert:

Erdgeschoß: in allen Räumen im Fußboden S-Matte Dachgeschoß: Kinder- und Schlafzimmer

$\begin{array}{ll}\begin{array}{l}\text { in Wand, Dachschräge und Decke } \\ \text { Bad im Fußboden }\end{array} & \begin{array}{l}\text { G-Matte } \\ \text { S-Matte }\end{array} \\ \text { Flur in der Wand } & \text { G-Matte } \\ \text { direkt unter den Dachziegeln } & \text { S-Matte }\end{array}$

Dach: direkt unter den Dachziegeln G-Matte

Das Kapillarrohrmattensystem läßt sich auch an ein vorhandenes Heizsystem (Öl, Gas usw.) anschließen, als separater Regelkreis mit Wärmetauscher und einem Mischventil zur Temperaturbegrenzung auf maximal $40{ }^{\circ} \mathrm{C}$, jedoch ohne Kiihlung.

\subsubsection{Saisonaler Erdspeicher}

Der saisonale Erdspeicher befindet sich im Bodenbereich des Hauses und ist in mehrere Speicherbereiche untergliedert (siehe Bild 5). Es ist ein Niedrigtemperaturspeicher, da seine Arbeitstemperatur bei maximal $30{ }^{\circ} \mathrm{C}$ liegt. [2]

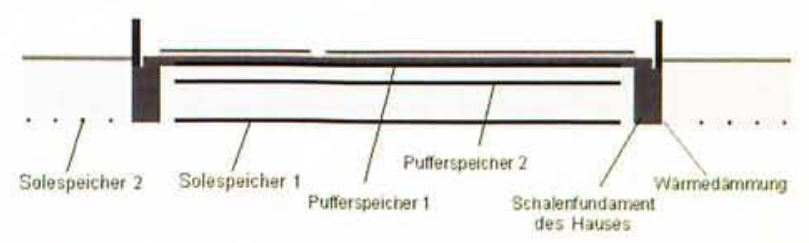

Bild 5: Querschnitt durch den Fundamentbereich des Hauses mit verschiedenen Speicherbereichen

Für Heizungszwecke muß der gesamte Erdspeicher mit einer Wärmepumpe gekoppelt werden. Der Wärmetransport erfolgt durch ein Wasser-Glykol-Gemisch in Polyäthylenschläuchen von $40 \mathrm{~mm}$ Querschnitt in vier separaten Kreisen. Pufferspeicher 1 befindet sich direkt in der Fundamentplatte, Pufferspeicher 2 ca. $35 \mathrm{~cm}$ tiefer in der Aufschüttung. Hier soll die überschüssige Wärme der Solarflachkollektoren zwischengespeichert werden. Sie kann bei Heizbedarf wieder abgenommen werden, entweder direkt oder mit Unterstüitzung der Wärmepumpe. 
Die Solespeicher 1 und 2 befinden sich in ca. $1 \mathrm{~m}$ Tiefe, der Solespeicher 1 unterhalb des Hauses und Solespeicher 2 außerhalb der Hausfläche. In diesen oberflächennahen Bereichen wird die Temperatur des Erdreiches wesentlich durch solare Einstrahlung, Abstrahlung, Konvektion, Niederschläge und nur gering durch Wärmeleitung aus dem Erdinneren bestimmt. Eine völlige Entkopplung von den atmosphärischen Vorgängen ist erst unterhalb $10 \mathrm{~m}$ Tiefe zu erwarten. Damit ist die Temperatur des Erdreiches zwar an die jeweilige Außentemperatur gekoppelt, eine ausreichende Pufferung ist jedoch vorhanden. Im Winter liegt die Temperatur des Bodens überwiegend oberhalb der Lufttemperatur, so daß das Erdreich als Wärmereservoir für eine Wärmepumpe genutzt wird. Im Sommer liegt die Bodentemperatur überwiegend unterhalb der Lufttemperatur (Wärmesenke) und wird direkt zum Kühlen der Räume genutzt.[3] [4]

\subsubsection{Flachkollektor und Wärmepumpe}

Zur Unterstützung der Gewinnung thermischer Solarenergie sind auf der Westseite des Daches vier Flachkollektoren (UFE SOLAR Eurostar SC 24) installiert worden. Jeder Kollektor hat eine Absorberfläche von $2,1 \mathrm{~m}^{2}$.

Die eingesetzte Wärmepumpe der Firma Turbovent Schweden arbeitet mit einer Leistungsaufnahme von 1,4 kW und einer -abgabe von $8,0 \mathrm{~kW}$. Die Leistungszahl beträgt unter Normbedingungen 4,5.

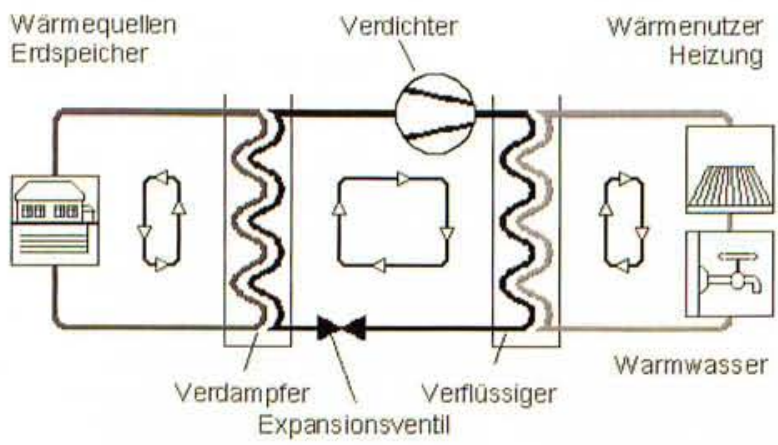

Bild 6: Prinzipieller Aufbau der Wärmepumpenanlage

Die Wärme, die sich auf einem unteren Temperaturniveau (Erdspeicher) befindet, wird durch Zufuhr von Energie auf ein höheres Temperaturniveau gebracht. Im Verdampfer entzieht das Arbeitsmittel des Kreisprozesses dem durch eine Pumpe geförderten Massenstrom der Wärmequelle (Erdspeicher) die zur Verdampfung notwendige Wärme. Der Verdichter saugt die Dämpfe aus dem Verdampfer ab und erhöht den Druck sowie die Temperatur unter Einwirkung von elektrischer Energie. Im Verflüssiger kondensieren die erhitzten Dämpfe und geben dabei die dem Erdspeicher entzogene Wärme an die Wärmenutzungsanlage (Heizung und Warmwasser) ab. [5]

\subsection{Energiemanagement}

\subsubsection{Jahreszeitliche Abhängigkeit der Betriebszustände} Das Betriebsjahr wird in zwei Perioden eingeteilt, die folgende Betriebszustände aufweisen (Tafel 1):

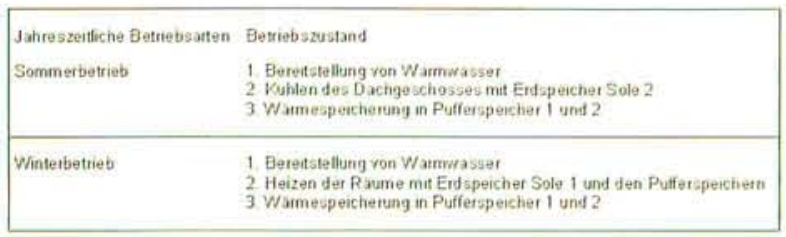

Tafel 1: Betriebszustände in einem Betriebsjahr

Das solare Energieangebot wird über das gesamte Jahr genutzt. Flachkollektoren und Kapillarrohrmatten nehmen die Wärme auf. Je nach Bedarf und Energieniveau wird sie verteilt bzw. gespeichert. Liegt die in den Kollektoren erreichte Temperatur unter dem Bedarfsniveau, aber über einem Speicherniveau, so kann diese Wärmeenergie dem entsprechenden Speicher zugeführt werden und steht der Wärmepumpe zur Verfügung.

Aus der Vielzahl der regelungstechnisch möglichen Zustände, werden die Hauptarbeitsregime im folgenden beschrieben. (vgl. Bilder 7 bis 10, aus Gründen der Übersichtlichkeit sind jeweils nur die in Betrieb befindlichen Teilkreise dargestellt)

\subsubsection{Wärmespeicherung}

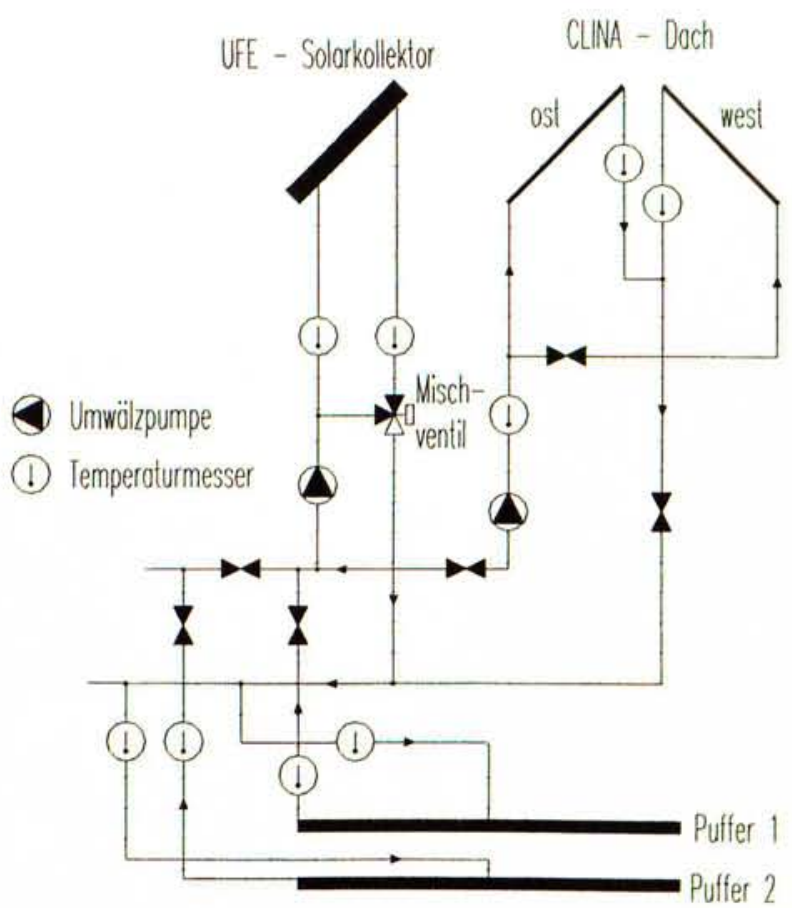

Bild 7: Schema der Wärmegewinnung mit Solarkollektoren und Kapillarrohrmatten

Flachkollektoren und Kapillarrohrmatten im Dach nehmen insbesondere in den Sommermonaten mehr Wärme von der Sonne auf als im Hausbetrieb benötigt wird. Sie wird in den Pufferspeichern 1 und 2 zwischengespeichert und steht dem System jederzeit zur Verfuigung (s. Bild 7).

\subsubsection{Warmwasserbereitung und Heizen}

Das Regelungssystem ist auf Warmwasservorrang ausgelegt, d. h. im Sommer wie im Winter wird das vorgewählte Temperaturniveau des Brauchwassers aufrechterhalten. Das Schema der Brauchwassererwärmung über das System Wärmepumpe/Wärmetauscher und die Sensorik ist im Bild 8 dargestellt. 


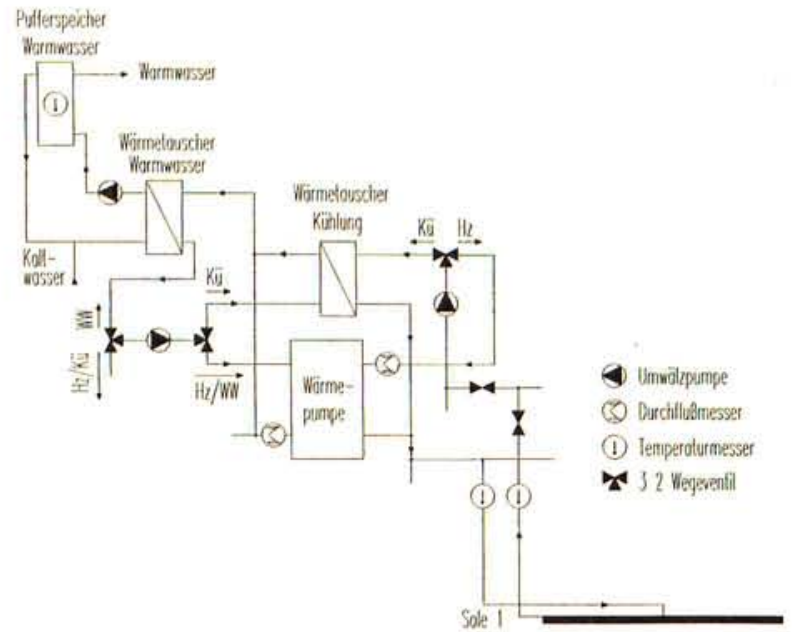

Bild 8: Schema der Brauchwassererwärmung

Wird keine Energie für die Warmwasserbereitung benötigt, kann diese bei Bedarf für die Raumheizung verwendet werden. Bild 9 zeigt das entsprechende Schema einschließlich der Meßstellen. In jedem Raum ist ein Thermostat installiert, das eine individuelle Raumtemperierung ermöglicht. Die Heizkreisverteiler regulieren den Wärmeträgerstrom. Der „Pufferspeicher Heizen“ ist aufgrund der Betriebsenergieversorgung der Wärmepumpe notwendig. Diese wird vom Energieversorgungsunternehmen zweimal pro Tag für eine Stunde abgeschaltet. In dieser Zeit wird die benötigte Wämeenergie aus diesem Pufferspeicher entnommen.

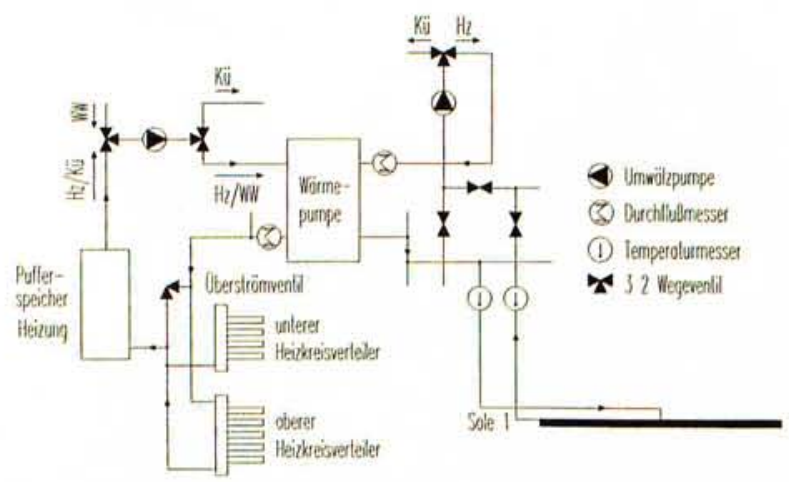

Bild 9: Schema der Gebäudeheizung

\subsubsection{Kühlen}

Um Kondenswasserbildung zu vermeiden, ist im größten Raum des Dachgeschosses ein Taupunktfühler installiert. Dieser signalisiert eine Taupunktunterschreitung

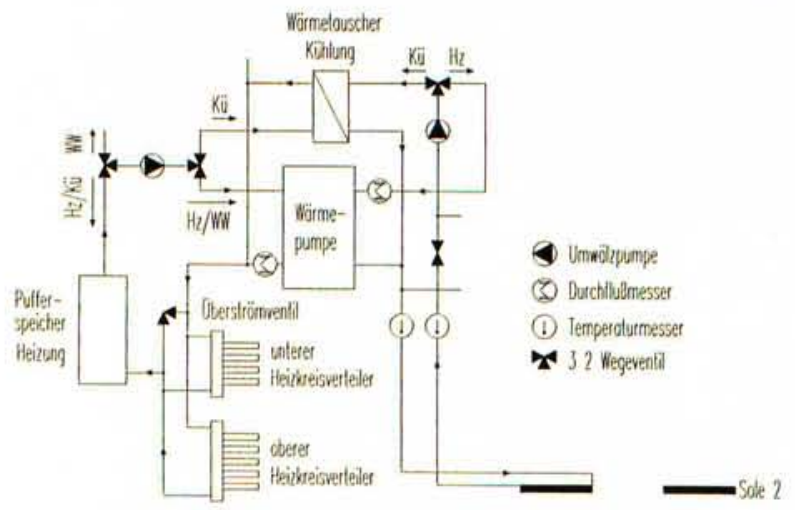

Bild 10: Schema der Kühlung des Dachgeschosses und veranlaßt die Regelung die Raumkühlung einzustellen bis die Kondensation der in der Luft befindlichen Feuchtigkeit an den zu kühlen Wänden aufhört.

\subsubsection{Meßdatenerfassung und Auswertung}

Zur detaillierten Einschätzung der Wirksamkeit der einzelnen Betriebszustände der Regelung wie auch des Gesamtverhaltens des Systems „Haus“ soll ein umfangreiches Meßsystem installiert werden. Um die verschiedenen in dieser Pilotanlage enthaltenen Komponenten in ihrem funktionellen Zusammenspiel in den jeweiligen Betriebszuständen zu optimieren ist eine Meßwerterfassung vorgesehen. An den erforderlichen Meßstellen im Fundamentbereich, welche nicht mehr zugänglich sind, wurde die Sensorik bereits eingebaut (siehe Bild 11).

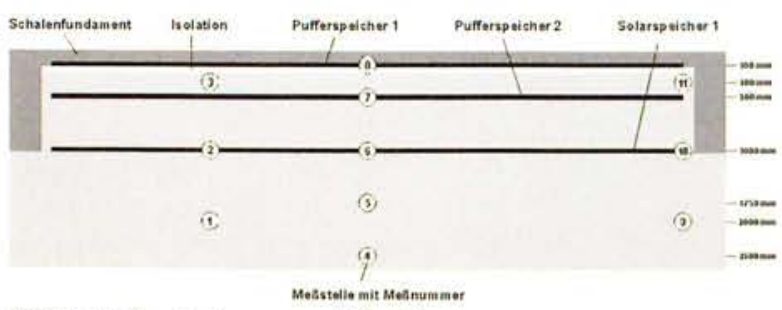

Bild 11: Meßwerterfassung am Erdspeicher

Gegenwärtig werden die restlichen Temperaturfühler und Durchflußmesser in den Räumen, sowie die Strahlungssensorik im Dachbereich installiert und durch Meßboxen der Firma L \& P Electronic GmbH aus Frankfurt/Oder ergänzt. Die Datenaufzeichnung und -verwaltung, die grafische Darstellung der Meßwerte und -ergebnisse sowie die Auswertung erfolgen über einen Personalcomputer. Auf der Grundlage der erhaltenen Daten soll eine Modellbildung insbesondere des Verhaltens der Erdspeicher ermöglicht werden. Ziel ist es, über die Simulation von Anlagenstrukturen ein Planungsinstrument für derartige Enegiesysteme zu erhalten und die jeweiligen Regelungsaufgaben zu optimieren.

\section{Wirtschaftlichkeitsbetrachtung}

Im Mittelpunkt steht das günstige Systemverhalten durch den Einsatz der Kapillarrohrmatten. Sie weisen eine große spezifische Oberfläche auf und ermöglichen geringe Vorlauftemperaturen beim Heizen von Räumen. Damit werden auch Energiequellen mit niedrigen Energie-(Temperatur-)niveaus interessant. Der Einsatz einer Wärmepumpe bietet sich an. Die Möglichkeit der Raumkühlung mit einfachster, praktisch wartungsfreier Anlagentechnik ist ein weiterer Aspekt, der hier nur qualitativ berüicksichtigt wird.

Für diese Wirtschaftlichkeitsbetrachtung soll der Vergleich mit einer klassischen Fußbodenheizung auf Gasbasis herangezogen werden (siehe Tafel 2). 


\begin{tabular}{|c|c|c|}
\hline Kntenum & \begin{tabular}{|l|} 
Gasheizung, \\
Komvektortheizkomet
\end{tabular} & $\begin{array}{l}\text { Kapillantohimatten system, } \\
\text { Waimepumpe (SOL ARKOON) }\end{array}$ \\
\hline \multicolumn{3}{|l|}{ Ausstattung/ Ouabitat } \\
\hline Fulsbodanheirung & Zusate & Standard \\
\hline $\begin{array}{l}\text { Strahlung sheizllachen } \\
\text { in Whand oder Decken }\end{array}$ & $\begin{array}{l}\text { nicht vorgesehen } \\
\text { bzw nichi mondich }\end{array}$ & Standard \\
\hline $\begin{array}{l}\text { Kuhlung im System } \\
\text { aber Decke oder Wand }\end{array}$ & nicht moglich & Standard \\
\hline $\begin{array}{l}\text { Brauciwwassererwatmung } \\
\text { zu } 60 \% \text { uber Solarenergie }\end{array}$ & Aulpteis ca 8.500, DM & Aufpreis ca. $4.500 .-D M$ \\
\hline $\begin{array}{l}\text { Heizungsunterstutzung } \\
2025 \% \text { uber Solarenergie }\end{array}$ & Aufpreis ca. $25000,-D M$ & Aufpreis ca 8.500, DM \\
\hline Halthatkes Heizkomet/flichen & 10. 15 Jahre & 50 - 70 Jahre \\
\hline Haltbarket Warmearzeuger & 8. 10 Jahie & 15 Jahre \\
\hline Wartunusntervall & Jahrfich & 3. 5 Jahte \\
\hline
\end{tabular}

Heizkostenschatzung Nevibu $130 \mathrm{~m}^{2}$ Wohnfllache

\begin{tabular}{|l|c|c|}
\hline Warmebedarf Heizung & $17000 \mathrm{kWh}$ & $15000 \mathrm{WWh}$ \\
\hline Warmebedarf Warmwasser & $2000 \mathrm{kWh}$ & $2000 \mathrm{WWh}$ \\
\hline Erdwarme & & $12750 \mathrm{kWh}$ \\
\hline Energieverbrauch & $27143 \mathrm{KWh}$ & $4250 \mathrm{WWh}$ \\
\hline Energiekasten pro Jahr & $1929 \mathrm{dM}$ & $620 \mathrm{DM}$ \\
\hline CO,-Emission & $5157 \mathrm{~kg}$ & $646 \mathrm{~kg}$ \\
\hline
\end{tabular}

Systemkosten

\begin{tabular}{|c|c|c|}
\hline Inyestitionssumme & $195000 \mathrm{M}$ & $31 \mathrm{DOO} D \mathrm{~A}$ \\
\hline Zinssatz (10 Jahre foat) & $7.20 \%$ & $4,86 \%$ (gefordent) \\
\hline Laufzeit & 29 Jahre & 20 Jahre \\
\hline Zims und Tilgung pro Jaht & $1599 \mathrm{DM}$ & $1910 \mathrm{DM}$ \\
\hline Heizkosten pro Jahr & $1929 \mathrm{DM}$ & $520 \mathrm{DPA}$ \\
\hline Wartung pin Jahr & $350 \mathrm{DM}$ & $150 \mathrm{DM}$ \\
\hline Schornstenfeger pro Jahr & $150 \mathrm{DM}$ & 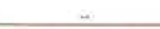 \\
\hline Förderung & - & 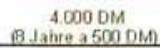 \\
\hline Gesamtausqaben pro Jahr & $3998 \mathrm{DM}$ & $21800 \mathrm{DM}$ \\
\hline
\end{tabular}

Tafel 2: Vergleich einer konventionellen Fußbodenheizung mit der SOLARKON Heizung

\section{Zusammenfassung}

In diesem Pilotprojekt für das Wärmemanagement im Wohnbereich ist ein Kapillarrohrmattensystem mit Wärmepumpe, Erdspeicher und Solarkollektor zum Einsatz gekommen. Als Wärmetauscher eingesetzt können mittels des Kapillarrohrmattensystems Räume ohne größeren technischen Aufwand im Winter beheizt und im Sommer gekühlt werden.

Durch die vorteilhaften Abmessungen des Kapillarrohrmattensystems ergibt sich ein sehr günstiges Verhältnis von Wärmeïbergangsfläche zu bedeckter Gesamtfläche bei minimaler Baudicke, d. h. das System zeichnet sich durch eine hohe Leistungsdichte aus. Damit gelingt es, die Nutzung der Erdwärme sowie auch einen geringeren Wärmeeintrag bei Unterstützung durch eine Wärmepumpe wirtschaftlich zu gestalten.

Die Flexibilität und die geringe Einbauhöhe erleichtern den Einbau des Mattensystems. Da sich die Kapillarrohrmatten im Fußboden-, Wand- und Deckenbereich sowie in den Dachschrägen befestigen lassen, ist die Installation nicht nur an einen bestimmten Ort im Raum gebunden.

Nach Abschluß der Installationsarbeiten für die Meßund Auswertungstechnik soll eine computergestützte Optimierung sowie die Simulation und die Modellierung von modifizierten Anlagen ermöglicht werden.

Wirtschaftlichkeitsbetrachtungen zeigen, daß die vorgestellte Anlage durchaus konkurrenzfähig ist. Die An- schaffungskosten sind zwar gegenüber anderen Heizsystemen etwas höher, man sollte jedoch nicht außer acht lassen, daß die jährlichen Betriebskosten für Schornstein, Brennstoff usw. entfallen.

Als Marktvorteile dürften sich erweisen, daß sich ganzjährig ein behagliches Raumklima einstellen läßt und die gesamte Anlage schadstofffrei sowie nahezu wartungsfrei arbeitet.

\section{Literaturverzeichnis}

[1] K. Heinloth, Die Energiefrage, Handbuch Umweltwissenschaften, 1. Auflage, Friedr. Vieweg \& Sohn Verlagsgesellschaft mbH, Braunschweig/Wiesbaden 1997

[2] H. Schulz, Wärme aus Sonne und Erde, ökobuch Verlag, 3. uiberarbeitete und erweiterte Auflage, Staufen 1995

[3] V. Quasching, Regenerative Energiesysteme, TechnologieBerechnung- Simulation, 1. Auflage, Carl Hanser Verlag München Wien 1998

[4] H. Elhasnaoui, Design of a Central Solar Heating Plant with Seasonal Storage - a Thesis Outline University of Massachussetts 1990

[5] H. Kirn, Wärmepumpen, Band 5: Wärmequellen und Wärmespeicher, 1. Auflage, Verlag C. F. Müller GmbH, Karlsruhe 1983

\section{Verfasser}

Prof. Dr. rer. nat. Siegfried Rolle

Dipl.-Ing. Michael Jergović

Dipl.-Ing. Henner Hoppe

Technische Fachhochschule Wildau

Fachbereich Ingenieurwesen/Wirtschaftsingenieurwesen Tel. (0 33 75) 507-145

Dipl.-Ing. (FH) Roy-Oliver Adams Dipl.-Ing. (FH) Marko Friedemann SOLARKON GmbH i.G.

16230 Blütenberg

Tel. (0 33 34) 59151 